\title{
Correcting misperceptions of relative income: impact on temporal discounting and social trust.
}

\author{
Léonard Guillou ${ }^{1}$, Aurore Grandin ${ }^{2}$, and Coralie Chevallier ${ }^{2}$ \\ ${ }^{1}$ Institut Jean Nicod, Département d'études cognitives, ENS, EHESS, CNRS, PSL Research \\ University, 75005 Paris, France \\ ${ }^{2}$ Laboratoire de neurosciences cognitives et computationnelles, Département d'études cognitives, \\ École normale supérieure, INSERM U960
}

May 2020

\begin{abstract}
Social trust and income are associated both within and across countries, such that higher income typically correlates with increased trust. While this correlation is well-documented, the psychological mechanisms sustaining this relationship remain poorly understood. One plausible candidate is people's temporal discounting: on the one hand, trust has a strong time component - it exposes the individual to immediate costs in exchange of uncertain and delayed benefits; on the other hand, temporal discounting is robustly influenced by income. The goal of our studies was to test whether temporal discounting mediates the relationship between income and trust and whether experimentally manipulating perceived income has a downstream impact on temporal discounting and trust. To do so, participants underestimating their relative income position were randomized to receiving information about their true position in the income distribution or no information. In line with our hypothesis, our results indicate that temporal discounting mediates the effect of income on social trust in a pre-registered online study on British participants $(\mathrm{N}=855)$. However, receiving a positive information shock on one's income position had no impact on either temporal discounting or social trust. In a second pre-registered study, we replicated the finding that temporal discounting mediates the effect of income on social trust in a representative sample of the British population $(\mathrm{N}=1130)$.
\end{abstract}




\section{Keywords}

Temporal discounting; perceived relative affluence; social trust; information shock.

\section{Introduction}

Social trust can be defined as the belief that other people are reliable cooperation partners. When one decides to take part in a cooperative interaction, it makes sense to invest in the interaction to the extent that one can trust that the cooperation partner will provide an adequate return on investment. This return can take the form of later reciprocation or of a reputational gain. Formally then, social trust is the belief that most people will reciprocate offer a return on investment - if one invests in a social interaction with them.

Social trust is a belief central to decision-making processes and it affects a range of prosocial behaviors like cooperation in situations of conflict of interests (Balliet and Van Lange, 2013), acceptance of vaccination (Rönnerstrand, 2013), or cooperation in social dilemmas with a large number of actors (Mannemar Sønderskov, 2011). At the societal level, social trust is linked to a host of positive outcomes, such as improved economic growth (Bjørnskov, 2017; Knack and Keefer, 1997). But despite its positive outcomes, social trust varies considerably between individuals and societies (Albanese and Blasio, 2014). Why is this the case? Do variations in social trust follow a predictable pattern? Can these variations be traced back to specific psychological and environmental features?

One factor explaining the variability of social trust is that it is sensitive to socioeconomic status. Existing works suggest that higher childhood socioeconomic circumstances (Mell et al., 2020; Stamos et al., 2019) as well as current income (Alesina and La Ferrara, 2002) are positively associated with social trust. Brandt et al. (2015) also showed with longitudinal data, that increases in socioeconomic status predict increases in social trust, and that this model is more viable than a model using increases in social trust to predict increases in socioeconomic status. Similarly, exogenous negative shocks on childhood resources have an impact on adult levels of social trust. Using data from Germany after the second World War, Hörl et al. (2016) showed that children exposed to more hunger during childhood had lower levels of trust as adults. Petersen and Aarøe (2015) even found an effect of lower birth weight on social trust in adulthood.

While the association between social trust and socioeconomic status is robustly established, the psychological mechanism by which this takes place 
is not known. One possibility is that social trust reacts to individual variations in temporal preferences, which are themselves affected by socioeconomic status. Social trust is indeed associated with certain and immediate costs and with uncertain and delayed benefits. People with short-term preferences should therefore be less prone to investing in social interactions, for which the return on investment is delayed. In terms of behaviors, the trade-off would be between choosing the sooner smaller reward of defecting over the later larger reward offered by reciprocation from the interaction partner or a reputational gain. In a recent paper, Pepper and Nettle (2017) suggested that the varying length of time-horizon between individuals could explain several socioeconomic gradients in social behaviours, including financial decisions (Falk et al., 2015; Howlett et al., 2008), educational attainment (Falk et al., 2015), academic engagement and performance (Brown and Jones, 2004), smoking (Adams, 2009) or eating behaviors (Adams and Nettle, 2009). We apply this logic to social trust: our first hypothesis is that temporal discounting is a key psychological mechanism by which socioeconomic status impacts social trust. In other words, we hypothesize that temporal discounting mediates the relationship between socioeconomic status and social trust.

The link between temporal discounting and social trust has only been tested in a couple of studies, with mixed findings. A first study, conducted in Vietnam, did not show any effect of temporal discounting on trust, using a trust game (Nguyen et al., 2012) but this null effect might be due to a lack of statistical power ( $\mathrm{N}=156$ only). A second study found that temporal discounting is an important covariate of self-reported trust (Albanese et al., 2017) but in this study, temporal discounting was measured with a single question, which limits the accuracy of the temporal discounting estimate. Our experimental design tackles both limitations and allows us to test whether variations in temporal discounting are associated with variations in social trust inside a mediation model. To avoid the pitfalls of the two studies above-mentioned we used well-validated measures of time discounting and social trust and we made sure to have sufficient power.

Moving beyond correlations, the goal of our paper is also to study the causal structure of this association by experimentally manipulating perceived income, which is a well-known driver of temporal discounting. In this context, our second hypothesis is that experimentally inducing fluctuations in temporal discounting should impact social trust.

Socioeconomic factors are associated with variability of discount rates between individuals (Green et al., 1996; Harrison et al., 2002; Reimers et al., 2009). Temporal discounting is typically measured using delay discounting experiments in which participants are asked to choose between a smaller but sooner reward - for example $\$ 2$ tomorrow - and a larger later reward - for 
example $\$ 5$ in one week. In these types of experiments, lower socioeconomic status individuals consistently display steeper temporal discounting (Green et al., 1996; Harrison et al., 2002; Reimers et al., 2009). Less educated adolescents, compared to adolescents with higher education, also show the same pattern (Lee et al., 2013). Interestingly, there is also evidence that experimentally induced income shocks have an impact on temporal discounting. Haushofer et al. (2013) for instance, showed that participants who received a negative income shock on the money earned from a real effort task, displayed increased discount rates. More recently, other studies have also found that negative income shocks increase delay discounting (Bickel et al., 2016; Mellis et al., 2018).

To manipulate socioeconomic status, we capitalise on work demonstrating that many people underestimate their wealth and that it is possible to correct these misperceptions, with measurable effects on attitudes and behaviours. For instance, faulty beliefs about inequalities are common in the US (Norton and Ariely, 2011) or in Australia (Norton et al., 2014). In both countries, people underestimate the level of wealth inequality in their country. The same occurs for one's own position in the income distribution, for example in Sweden (Karadja et al., 2014) or in Argentina (Cruces et al., 2011). In these countries, people underestimate their relative position in their country's income distribution. These misperceptions can be explained by people's overreliance on cues from their local environment (Hauser and Norton, 2017). Interestingly, studies have shown that providing economic information (for instance, about inequalities or income distribution) as an experimental treatment can have an impact on people's attitudes, e.g. their preference for redistribution (Cruces et al., 2011; Karadja et al., 2014) or tolerance for inequalities (Kuziemko et al., 2013). In this paper we apply a similar method in order to test whether experimentally manipulating perceived income has a downstream impact on temporal discounting and trust.

To test our hypotheses we designed two online studies. In study 1 , we tested a causal mediation model with time discounting mediating the effect of socioeconomic status on social trust. To do so, we used an experimental design by randomly informing a subsample of participants about their true relative income position in the British society. Since objective socioeconomic status cannot be easily manipulated experimentally (except by giving people extra income), we focused on the way people perceive their resource level (i.e. their perceived relative income). Specifically, we corrected people's perception of their income when they felt that their relative income position was lower than it actually was. In other words, the goal of our intervention was to provide a "positive psychological income shock". Our main question was whether correcting people's misperception of their rel- 
ative income would influence temporal discounting and as a consequence, social trust. We hypothesized that respondents who learnt that they had a higher relative income than they thought (positive income shock) would discount future benefits less, and that as a result they would display more social trust. The experiment targeted only participants who hold wrongly negative beliefs about their position in society. For ethical reasons, participants who believed they had a higher relative income than they actually had, did not receive any correction. We also studied this mediation pathway with a correlational approach testing if the effect of being of low or high socioeconomic status on social trust was mediated by temporal discounting.

In study 2 we replicated our results from study 1, particularly the mediating role of temporal discounting on the relationship between socioeconomic status and social trust and between time discounting and social trust. In this study we did not test any treatment, data were only observational. This replication was made on a sample representative of the British population, which was not the case in study 1.

\section{$1 \quad$ Study 1}

In this study, we tested whether temporal discounting mediates the relationship between income and trust and we tested the causal influence of perceived relative income on people's temporal discounting and social trust. To do so, we used an experimental design by randomly informing a subsample of participants about their true relative income position in the British society. Our main question was whether this income shock about participants' actual relative income could influence temporal discounting and as a consequence, social trust. We hypothesized that respondents who learnt that they were underestimating their relative income (and who thus benefited from a positive income shock) would discount future benefits less, and that as a result they would display more social trust. The experiment targeted only participants who wrongly held negative beliefs about their relative income. For ethical reasons, participants who overestimated their relative income did not receive any correction.

\section{$1.1 \quad$ Methods}

\subsubsection{Participants}

Participants were recruited online via Prolific Academic (https://www. prolific.co). We used Prolific's pre-screening criteria to filter participants by nationality and approval rate. We recruited only British participants with 
a minimum approval rate of $90 \%$. Participants were excluded if they reported a personal monthly income above $£ 12,500$, which corresponds to the highest tax bracket and top earning percentile in the UK, in order to minimize potential income reporting mistakes. We also excluded students because their reported income may not reflect their actual living standards (e.g., students may report no income but receive parental support).

To determine sample size, we set an a priori power level and a minimum detectable effect that the intervention was expected to have on temporal discounting (Djimeu and Houndolo, 2016). We used the formula recommended by Bloom (2008): $M D E=\left(t_{1-k}+t_{\alpha / 2}\right) * \sqrt{\frac{1}{P(1-P)}} * \sqrt{\frac{\sigma^{2}}{N)}}$. Where MDE $=$ Minimum Detectable Effect; $\mathrm{N}=$ Number of observations; $\sigma=$ standard deviation of effect; $\mathrm{P}=$ proportion of treated $(50 \%) ; t_{\alpha / 2}=1.96$ (using a two-tailed hypothesis at the .05 significance level); $t_{1-k}=0.84$ (setting statistical power to $80 \%$ ). We based our power calculation on measures of temporal discounting taken in a British sample (Grandin et al., in prep, see pre-registration: https://osf.io/vq8t3). We found that we needed 807 participants to identify a standardized minimum detectable effect of 0.2 . To compensate for attrition, we recruited 888 participants, which corresponds to 807 plus $10 \%$ attrition. The study used for the power analysis (Grandin et al., in prep, see pre-registration: https://osf .io/vq8t3) did not contain any question on social trust. Therefore, we had no means of knowing whether a study with 888 participants was sufficiently powered to detect an effect of an income shock on social trust mediated by temporal discounting.

\subsubsection{Materials}

The questionnaires and discounting task were presented using Qualtrics (https://www.qualtrics.com).

Socioeconomic status. We included two measures of socioeconomic status: an objective measure and a subjective measure. Objective socioeconomic status refers to income and education. We asked respondents about their personal income rather than their household overall income, because studies have shown that questions about household income induce lower response rates and produce lower quality data (Micklewright and Schnepf, 2010). Participants were asked to report their personal monthly income in a free-text box in order to avoid unintentional priming effects that come with the use of income brackets (Haisley et al., 2008). Moreover, in order to minimize reporting mistakes such as reporting yearly instead of monthly income, or unintentionally typing an additional zero, we calculated their annual income based on the monthly income they reported and asked them to confirm that it corresponded to their actual earnings. In addition to personal income, we 
asked participants to specify which amount of benefits they received (jobseeker's allowance, incapacity benefits, etc.).

We acknowledge that it is not only income or occupation that generate differences in behaviour, but the experience of various deprivations that are often associated with being of lower socioeconomic status (Pepper and Nettle, 2017). Therefore, we also computed a measure of perceived relative affluence (subjective socioeconomic status) combining the MacArthur Scale of Subjective Social Status (Adler et al., 2000) and Griskevicius et al. (2013)' three-item questionnaire.

We used a slightly modified version of the MacArthur scale in order to focus on income: "Think of this scale as representing where people stand in the United Kingdom. At the top of the scale (10) are the people who are the best off in terms of overall income. At the bottom (1) are the people who are the worst off in terms of overall income. Where would you place yourself in this scale?" Griskevicius et al. (2013)'s three-item questionnaire was: "How much do you agree with each of the below statements on a scale from 1 (strongly disagree) to 7 (strongly agree)? My family and I have had enough money for things. I have lived in a relatively wealthy neighborhood. I have felt relatively wealthy compared to other people in my neighborhood."

Temporal discounting. Prior research has demonstrated that hypothetical delay-discounting tasks reliably predict actual temporal preferences (Hardisty et al., 2013), and correlate with real-world measures of impulsivity such as smoking, overeating, and credit card debt (Chabris et al., 2008; Meier and Sprenger, 2009; Reimers et al., 2009). Our temporal discounting task was based on the one designed by Frye et al. (2016). In this task, participants had to complete three blocks of an intertemporal choice task with varying delays and amounts. All values displayed to participants were rounded to the nearest 50 pence (£0.50). Each block consisted of 6 binary choice trials. The task ended with two catch trials (e.g., "Do you prefer $£ 30$ in 3 days or $£ 1$ in 2 years?") to make sure that participants read carefully, resulting in a total of 20 trials. In the first block, participants had the choice between a smaller reward in three days, and a larger reward in three weeks. In the second and third blocks, the later delay was set to three months and two years respectively. The choices were hypothetical, which means that participants did not actually receive the money corresponding to their choices. As in Haushofer et al. (2013), possible serial correlations and order effects in participants' responses were controlled for by randomising the order of trials across blocks. In order to control for possible unintended effects, the position of the sooner-smaller and later-larger alternatives on the screen (top vs. bottom) was also randomised across trials. The later reward was kept constant at $£ 70$ (as in Reimers et al. (2009)), while the smaller reward was 
adjusted according to each participant's choices. The adjustment was based on a bisection algorithm, following Frye et al. (2016). As recommended by the authors, the adjustment for the upcoming trial was always equal to the maximum amount multiplied by $2^{-n}$, where $\mathrm{n}$ was the trial number for the current adjustment.

Such a procedure allowed us to identify three individual indifference points for each participant, which can be used as the basis for the computation of discount rates. In our case, the indifference point for a particular delay is the amount of money to which a participant is indifferent between receiving it in three days and receiving $£ 70$ with the particular delay. Given that we only have 3 indifference points per participant, we were not able to fit curvilinear regression models ((Green et al., 1996; Mazur, 1987; Rachlin, 2006)) and focused instead on the area under the curve (AUC) Frye et al. (2016). This measures carries partial information, because different curve shapes can result in the same area under the curve. We focused on AUC because our main goal is to estimate between-participants differences in temporal discounting steepness rather than to precisely model participants' discounting curves. Using area under the curve was therefore an optimal choice considering the trade-off between measurement precision and study duration.

Social Trust. Social trust is a complex behavior, which is difficult to fully capture in a brief study, but studies suggest that questionnaires are often better than economic games in predicting real-life behaviours (Galizzi and Navarro-Martinez, 2019; Laury and Taylor, 2008; Voors et al., 2012). Here, we focused on three questions that are routinely used in national and international surveys, such as the European Values Study: 'Generally speaking, would you say that most people can be trusted or that you can't be too careful in dealing with people?', 'Would you say that people usually only take care of themselves or that they try to be helpful most of the time?' and 'Do you think that most people would try to take advantage of you if they had the opportunity or that they would try to be fair?'. Answers available to participants for the first question were "most people can be trusted", "can't be too careful" or "don't know". A 1-10 scale was used for the second and third questions.

\subsubsection{Procedure}

Participants started with questions about socioeconomic status and demographics. We used these answers to calculate how much our participants underestimated or overestimated their relative position in the British income distribution. We calculated the sum of their reported income and taxable 
benefits to determine which decile they belonged to. We used statistics from the British government's Personal Income statistics release (https://www. gov.uk/government/collections/personal-incomes-statistics) as a reference to compute participants' position in the income distribution, which provides percentiles of total annual income (comprising taxable benefits). We compared this decile to their answer to the MacArthur scale, so as to estimate how much participants had a biased perception of their position in the income distribution. We defined the bias of a participant as the difference between her perceived and actual income decile. Participants who underestimated (/overestimated) their relative income by 1 decile point or more were categorized as having a negative (/positive) bias. The remaining participants were defined as having no bias. The survey stopped there for participants who estimated their position accurately or overestimated it (null or positive bias). Those who underestimated their position (negative bias) were randomly assigned to the experimental or control condition. In the experimental condition, participants were presented with a correction of their misperception of relative income, based on their reported income and taxable benefits. These participants therefore received a positive income shock. Participants then completed the temporal discounting task. Next they answered questions on environmental attitudes for another study. Questions on social trust came last.

\subsection{Data treatment}

All data treatment and analyses were carried out in R. They were all preregistered (see: https://osf.io/pyj24). The only deviation from the preregistration plan is that the exclusion for reaction times was applied at the single trial level. In what follows, we focus on the mediation analyses.

Exclusion of participants. Participants were excluded from all analyses if they failed one or more catch trials in the temporal discounting task. They were also excluded if they responded too fast $(<500 \mathrm{~ms})$ or too slowly $(>2$ minutes) to single question screens or if they responded too fast $(<3 \mathrm{~s})$ or too slowly ( $>5$ minutes) to the other survey pages (with more than one question per screen). We used these filters to make sure that participants were attentive and read the questions carefully. This left a sample of 855 participants with 442 women. Ages ranged 21 to $72, M=39.76, S D=10.46$.

Socioeconomic status. We computed total income, which was the sum of participants' personal monthly income and benefits. Education level was transformed from an unordered categorical variable (e.g., "Completed College") to a 1 to 6 score. Objective socioeconomic status was the sum of $\mathrm{z}$ scored total income and z-scored education level. Subjective socioeconomic 
status was the sum of z-scored items Griskevicius et al. (2013)'s questionnaire and MacArthur scale.

Information shock (treatment) corresponds to a binary variable indicating whether a participant was in the control group or the treated group.

Temporal discounting. To calculate individual temporal discount rates, we isolated the indifference point for each delay and for each participant and then calculated the area under the discounting curve linking these points. The AUC between two points on the curve is calculated as $\left(x_{2}-x_{1}\right)\left[\left(y_{1}+\right.\right.$ $\left.y_{2}\right) / 2$ ], where $x_{1}$ and $x_{2}$ are the successive delays and $y_{1}$ and $y_{2}$ are the indifference points for those delays. Then the AUC between 3 weeks and 3 months and the one between 3 months and 2 years were summed, resulting in a single value of AUC per participant. Lower values of AUC indicate steeper discounting (Frye et al., 2016). see Figure 1.

Social Trust. A general trust binary variable was constructed from the first question ('Generally speaking, would you say that most people can be trusted or that you can't be too careful in dealing with people?'), with a value of 1 for individuals responding "most people can be trusted" and a value of 0 for the others. A 1-10 scale was used for the two additional questions ("Would you say that people usually only take care of themselves or that they try to be helpful most of the time?' and 'Do you think that most people would try to take advantage of you if they had the opportunity or that they would try to be fair?'). The answers obtained to these three questions were z-scored and summed to obtain a single index capturing participants' beliefs on others' level of prosociality.

\subsection{Statistical analyses}

Correlational Mediation model. Our first main hypothesis (H1) is that the relationship between social trust and socioeconomic status is mediated by individual discount rates. To test the mediation model, we used the causalsteps procedure described by Baron and Kenny (1986). Following recommendations from Miller et al. (2007) and Aguinis et al. (2017), we tested the significance of the indirect pathway with the $\mathrm{R}$ mediation package (Tingley et al., 2014) using the function mediate. Despite the name of this package: "R Package for Causal Mediation Analysis", the mediation analysis described in this section is not causal because it is not based on a treatment but only on observational data. In the present case, the treatment variable (i.e., the "treat" argument in the mediate function) is socioeconomic status (alternatively objective or subjective).

Causal mediation model Our second main hypothesis (H2) is that the effect of the positive information shock on social trust is mediated by indi- 
vidual discount rates. To test this hypothesis we used the same steps as for H1 but with the binary variable "treatment" (0: control, 1: treated) as the treatment variable (i.e., the "treat" argument in the mediate function).

\subsection{Results}

For descriptive statistics, see Table 1.

For a graph of temporal discounting curves, see Figures 2 and 3.

Correlational Mediation model. To test our first main hypothesis (H1) we conducted regression analyses relying on the causal-steps procedure set forth by Baron and Kenny (1986) to detect a mediation effect. The results suggest a partial mediation: the effect size of both objective (see table 2) and subjective (see table 3) socioeconomic status is lowered by the addition of temporal discounting in the model. For both mediation pathways (Objective vs. Subjective socioeconomic status), the positive effect of socioeconomic status on social trust is partially mediated by temporal discounting (Objective socioeconomic status: Average Causal Mediating Effect of temporal discounting: $\beta=0.02, p=.001$, Proportion of the total effect that is mediated: $9.0 \%, p=.001$; Subjective socioeconomic status: Average Causal Mediating Effect of temporal discounting: $\beta=0.02, p=.01$, Proportion of the total effect that is mediated: $6.2 \%, p=.01$ ). These values are calculated for control.value $=-0.6$ (first quartile) and treat.value $=0.5$ (third quartile) for Objective socioeconomic status and control.value $=-0.7$ (first quartile) and treat.value $=0.7$ (third quartile). The Proportion of the total effect that is mediated and all the p-values do not vary if other control.value and treat.value are choosen, only $\beta$ values, change. See tables 4 and 5 .

Causal mediation model To test our second main hypothesis (H2) that the effect of the income shock on social trust is mediated by individual discount rates, we used the same steps as for $\mathrm{H} 1$ but with the binary variable "treatment" (0: control, 1: treated) as the treatment variable (i.e., the "treat" argument in the mediate function). The mediation analysis shows no direct effect of the income shock on social trust and no mediation (Average Causal Mediating Effect of temporal discounting: $\beta=0.001, p=.85$, Proportion of the total effect that is mediated: $-2.5 \%, p=.95)$. See table 6

\subsection{Discussion}

In this study, correcting participants' misperception of their relative income did not have any effect on their temporal discounting and social trust. There are several possible reasons explaining this outcome. First, participants may not have believed the information we provided. We specified that "the income 
statistics come from GOV.UK and are based on the whole British population above 18 (taxpayers only)" but this may not have been enough to convince participants that the information was accurate. Given that we did not ask a debriefing question to probe participants' confidence in the income correction, we have no way of knowing whether this was indeed an issue. Second, a correction of people's misperception of their relative income may not be a powerful enough treatment to affect temporal discounting or social trust. In line with this idea, Haushofer et al. (2013) found that negative income shocks had a larger effect on temporal discounting than positive income shocks. Karadja et al. (2014), on the other hand, did find an effect of positive income shocks but in a sample that was mostly negatively biased, unlike our sample, which was mostly positively biased. To sum up, we replicated the known correlations between socioeconomic status and temporal discounting on the one hand, and between socioeconomic status and social trust on the other hand. More interestingly we found that temporal discounting and social trust were correlated and that the effect of socioeconomic status on social trust was partially mediated by the temporal discounting. However, our study sample was very specific. Specifically, participants were excluded if they overestimated their relative income, which may limit the generalizability of our findings. Therefore, we decided to replicate our main results on a representative sample of the British population.

\section{Study 2}

In this study, our goal was to replicate the mediating effect of temporal discounting on the relationship between socioeconomic status and social trust, on a representative sample of the British population. The sample tested in Study 1 is indeed not representative because we only included participants who received an income and held wrongly negative beliefs about their relative affluence.

\section{$2.1 \quad$ Methods}

\subsubsection{Participants}

Participants were recruited online via Prolific Academic (https://www . prolific.co). Our final sample included a representative sample of 1188 participants, stratified across three demographics: age, sex and ethnicity via the representative sample option in Prolific Academic (including 10\% more participants for attrition). Sample size was determined a priori based on the 
results of study 1 with the "Monte Carlo Power Analysis for Indirect Effects" application (Schoemann et al., 2017).

\subsubsection{Materials}

We used the same materials as in study 1 . The questionnaires and discounting task were presented using Qualtrics (https://www.qualtrics.com).

\subsubsection{Procedure}

Participants started with questions about socioeconomic status and demographics. Then, they completed a temporal discounting task, and questions on social trust came last. All the measures were the same as those included in study 1.

\subsection{Data treatment}

All data treatment and analyses were carried out in $\mathrm{R}$ and were pre-registered (see pre-registration of study 1 https://osf.io/pyj24 and replication preregistration: https://osf.io/4cjkh). As in study 1, the only deviation from the pre-registered analysis plan is that the exclusion for reaction times was applied at the single trial level. As in study 1, we focus on the mediation analyses.

Exclusion of participants. The same criteria were applied as those in Study 1. This left a sample of 1130 participants with 585 women, 543 men and 2 others. Ages ranged 19 to 87, $M=45.56, S D=15.31$.

Temporal discounting. To calculate individual temporal discount rates, we followed the same method as in Study 1.

Social Trust. As in Study 1, the answers obtained to the 3 questions about social trust were z-scored and summed to obtain a single index capturing participants' beliefs on others' level of prosociality.

\subsection{Statistical analyses}

Correlational Mediation model. Our main hypothesis (H1) is that the relationship between social trust and socioeconomic status is mediated by individual discount rates. To test this hypothesis we conducted regression analyses relying on the causal-steps procedure set forth by Baron and Kenny (1986) to detect a mediation effect, following recommendations from Miller et al. (2007) and Aguinis et al. (2017). We tested the significance of the indirect pathway with R mediation package (see Tingley et al. (2014)) using 
the function mediate. As in study 1, we set two values (control.value and treat.value) of the treatment to make the contrast.

\subsection{Results}

For descriptive statistics, see table 7.

Correlational Mediation model. To test our main hypothesis (H1) we conducted regression analyses relying on the causal-steps procedure set forth by Baron and Kenny (1986) to detect a mediation effect. The results suggest a partial mediation: the effect size of both objective (see table 8) and subjective (see table 9) socioeconomic status is lowered by the addition of temporal discounting in the model. To test the significance of the mediation pathway we used R mediation package (Tingley et al., 2014) using the function mediate. We tested again two mediation pathways: one with Objective socioeconomic status and one with Subjective socioeconomic status. In both cases, the positive effect of socioeconomic status on social trust is partially mediated by temporal discounting (with Objective socioeconomic status: Average Causal Mediating Effect of temporal discounting: $\beta=0.04, p<.001$, Proportion of the total effect that is mediated: $28 \%, p=.002$; with Subjective socioeconomic status: Average Causal Mediating Effect of temporal discounting: $\beta=0.05, p<.001$, Proportion of the total effect that is mediated: $13 \%$, $p<.001$ ). These values are calculated for control.value $=-0.80$ (first quartile) and treat.value $=0.6$ (third quartile) for Objective socioeconomic status and control.value $=-0.73$ (first quartile) and treat.value $=0.70$ (third quartile) for Subjective socioeconomic status. The Proportion of the total effect that is mediated and all the p-values do not vary if other control.value and treat.value are choosen, only $\beta$ values, change. See tables 10 and 11 . The mediate function output labels these effects as "Causal Mediating Effect", but it is important to note that in our case, it is only correlational.

\subsection{Discussion}

Using a correlational mediation model we found that the relationship between socioeconomic status and social trust is mediated by individual discount rates. This replication on a sample stratified across three demographics: age, sex and ethnicity, confirms the results obtained in study 1. 


\section{General discussion}

The two studies reported in this paper investigate the link between socioeconomic status and social trust. Our goal was to identify a proximate psychological mechanism to explain this relationship. Our hypothesis was that temporal discounting plays an important role in the association between socioeconomic status and social trust. In line with this idea, we found a negative correlation between temporal discounting and social trust in two different samples, including a representative sample of the British population. In addition, we found that temporal discounting partially mediated the effect of socioeconomic status on social trust. The mediation scheme brought to light is an important step in the identification of proximate mechanisms explaining the relationship between socioeconomic status and behaviors like social trust.

The link between temporal discounting and social trust provides a new understanding of how social trust is modulated between individuals. It confirms the idea that social trust is modulated by long-term expectations and that it is therefore sensitive to temporal discounting. One possible explanation is that when someone has to decide whether or not to invest in a social interaction, their time preferences influence how much they are willing to wait in order to receive a return on their social investment. This is in line with the scientific literature linking temporal discounting and social behaviors, which suggests that several socioeconomic gradients in social behaviours can be traced back to variations in temporal preferences (Pepper and Nettle, 2017).

This study and its replication are the first ones combining a multi facet measure of socioeconomic status, a precise measure of temporal discounting (that involves more than a single choice), and a straight forward measure of social trust. In addition, both our studies are pre-registered, we made sure that we had sufficient power to test our hypotheses and we used a representative sample in the replication study. That is why our results make an important contribution to the limited literature on this topic (Albanese et al., 2017; Nguyen et al., 2012).

We also tried to alter temporal discounting using one of its most important predictors, socioeconomic status. We borrowed this idea from Karadja et al. (2014) but there is a growing literature looking at the impact of income and inequality perception on people's psychology. Some studies have focused on inequality perception in a company (Card et al., 2012) or in a given society (Bublitz, 2017; Cruces et al., 2011; Karadja et al., 2014) but political sciences have also investigated this topic (Fernández-Albertos and Kuo, 2018). These misperceptions are observed in many societies but are 
highly variable (Bublitz, 2017). Overall, correcting negative misperceptions seems to have less of an effect than correcting positive misperceptions. For instance, people who learn that they are relatively poorer than they thought demand higher levels of redistribution but people finding out that they are richer than they thought do not change their attitudes towards taxation and redistribution (Cruces et al., 2011).

Several limitations can explain the null effect obtained from the information shock on socioeconomic status provided to our participants in study 1. First our participants may have not believed us when we told them their real place in the income gradient of the British population. It is possible that other ways of informing participants may be more effective, for example showing them the true income distribution in their society and placing them on it (Bublitz, 2017; Kuziemko et al., 2013), or giving them a percentage of people earning less than them (Bublitz, 2017; Cruces et al., 2011; Kuziemko et al., 2013). The possibility that participants did not believe the information shock could also be linked to negativity bias. It has already been shown that negative information is often seen as truer that a positive one (Hilbig, 2009). In our case we only tested a positive information shock for ethical reasons but a negative shock might have been easier to believe for participants. Second, the treatment itself is perhaps not appropriate to impact temporal discounting and social trust. Temporal discounting and social trust are affected by childhood harshness (Mell et al., 2020; Stamos et al., 2019) and these long lasting effects may be a lot stronger than our information shock. People may need several repeated evidence of their true relative wealth on a longer timescale to express new temporal discounting.

Jachimowicz et al. (2017) have also studied another type of trust, community trust, in relation with socioeconomic status and temporal discounting. In their laboratory and field studies, they chose to focus on this particular kind of trust because community trust, unlike social trust (that they call generalized trust) is not correlated with socioeconomic status and is easier to target in interventions. They found that community trust can buffer the effect of low socioeconomic status on temporal discounting. Low income people with a high community trust displayed less temporal discounting than the ones with a low level of community trust. An individual with a high level of community trust may be more prone to believe that a later-larger reward will be offered by an other individual of its community. Or that the community will be able to provide help against a potential financial need in the meanwhile.

Further research could take several directions. In keeping with our study, it would be interesting to design new treatments that really impact participants' perception of relative affluence, for example, by using more convincing 
information shocks and checking if participants believe the experimenter. It could also be possible to focus on an other methods to shift temporal discounting and study the consequence on social trust. For instance, several studies have used episodic future thinking to modulate temporal discounting (Daniel et al., 2015; Liu et al., 2013; Sze et al., 2017). Another possible direction is to conduct longitudinal studies to compute time series analyses and test whether an exogenous increase in socioeconomic status is followed by a decrease of temporal discounting and then an increase in social trust. If an efficient treatment is found to shift people's perception of relative affluence or temporal discounting and if this consequently impacts social trust, the results would be of important interest for policy makers when designing interventions to promote prosocial behaviors.

\section{Figures \& Tables}

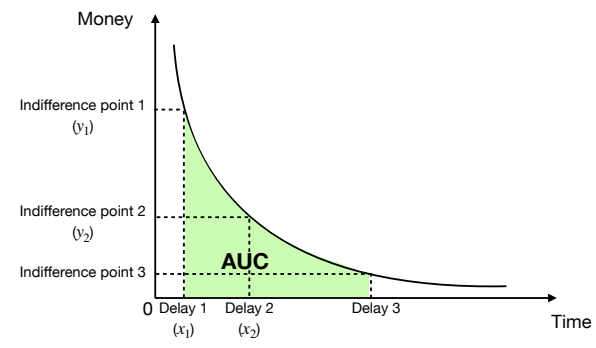

Figure 1: Temporal discounting curve. AUC: Area Under the Curve. In fact the AUC calculated is a little bit bigger than the theoretical one represented in green here because it is calculated as a trapezoidal area. 
Table 1: Descriptive statistics

\begin{tabular}{lcccc}
\hline \hline Statistic & Mean & St. Dev. & Min & Max \\
\hline Age (years) & 39.8 & 10.5 & 21 & 72 \\
Education score (1-6) & 4.1 & 0.9 & 1 & 5 \\
McArthur ladder (1-10) perceived & 5.2 & 1.5 & 1 & 9 \\
McArthur ladder correct & 7.5 & 1.8 & 2 & 10 \\
Total income (£) & $2,736.3$ & $1,257.6$ & 1,200 & 12,000 \\
AUC & $20,501.4$ & $12,934.1$ & 709.0 & $49,275.5$ \\
Total social trust & 0.00 & 2.5 & -5.4 & 5.7 \\
Griskevicius score & 12.7 & 3.3 & 3 & 21 \\
\hline
\end{tabular}

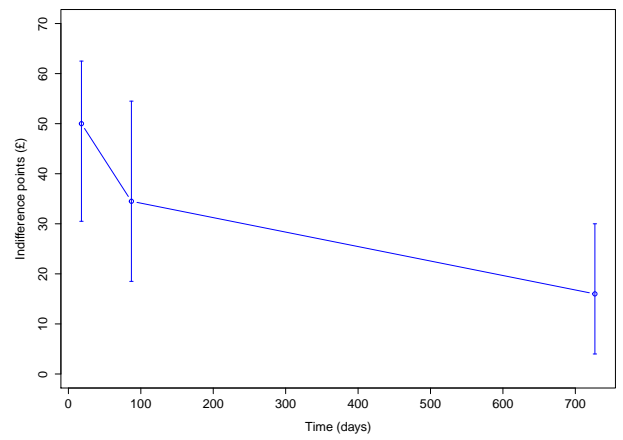

Figure 2: Medians of temporal discounting curves of the control group. The upper bound of error bars is the third quartile, the lower bound is the first quartile. The distance between them is the interquartile range. These three medians are significantly different (all $p<.001$ for paired Wilcoxon tests). This type of visualisation was chosen because indifference points are not normally distributed. 


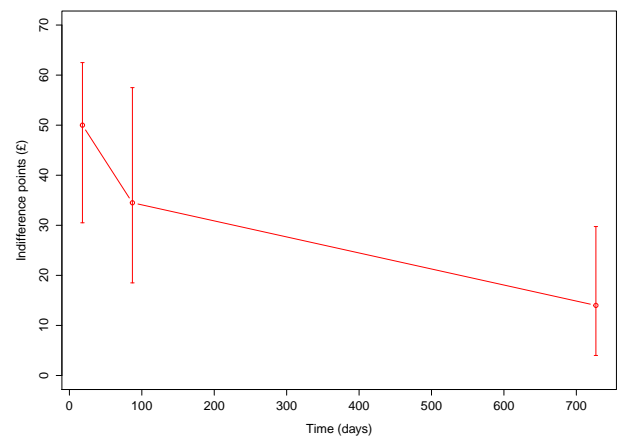

Figure 3: Medians of temporal discounting curves of the treated group. The upper bound of error bars is the third quartile, the lower bound is the first quartile. The distance between them is the interquartile range. These three medians are significantly different (all $p<.001$ for paired Wilcoxon tests). This type of visualisation was chosen because indifference points are not normally distributed

Table 2: Baron and Kenny (1986) steps procedure

\begin{tabular}{lccc}
\hline \hline & \multicolumn{3}{c}{ Dependent variable: } \\
\cline { 2 - 4 } & Total Trust & AUC & Total Trust \\
& $(1)$ & $(2)$ & $(3)$ \\
\hline Objective SES & $0.168^{* * *}$ & $0.135^{* * *}$ & $0.153^{* * *}$ \\
& $(0.034)$ & $(0.034)$ & $(0.034)$ \\
AUC & & & $0.111^{* *}$ \\
& & & $(0.034)$ \\
& & 855 & 855 \\
Observations & 855 & 0.018 & 0.040 \\
$\mathrm{R}^{2}$ & 0.028 & 0.017 & 0.038 \\
Adjusted $\mathrm{R}^{2}$ & 0.027 & $0.991(\mathrm{df}=853)$ & $0.981(\mathrm{df}=852)$ \\
Residual Std. Error & $0.986(\mathrm{df}=853)$ & $15.807^{* * *}(\mathrm{df}=1 ; 853)$ & $17.867^{* * *}(\mathrm{df}=2 ; 852)$ \\
F Statistic & $24.767^{* * *}(\mathrm{df}=1 ; 853)$ & & ${ }^{*} \mathrm{p}<.05 ;{ }^{* *} \mathrm{p}<.01 ;{ }^{* * *} \mathrm{p}<.001$ \\
\hline \hline Note: & & &
\end{tabular}


Table 3: Baron and Kenny (1986) steps procedure

\begin{tabular}{lccc}
\hline \hline & \multicolumn{3}{c}{ Dependent variable: } \\
\cline { 2 - 4 } & Total Trust & AUC & Total Trust \\
& $(1)$ & $(2)$ & $(3)$ \\
\hline Subjective SES & $0.264^{* * *}$ & $0.197^{* * *}$ & $0.248^{* * *}$ \\
& $(0.033)$ & $(0.034)$ & $(0.034)$ \\
AUC & & & $0.083^{*}$ \\
& & & $(0.034)$ \\
& & 855 & 855 \\
Observations & 855 & 0.039 & 0.076 \\
$\mathrm{R}^{2}$ & 0.070 & 0.038 & 0.074 \\
Adjusted $\mathrm{R}^{2}$ & 0.069 & $0.981(\mathrm{df}=853)$ & $0.962(\mathrm{df}=852)$ \\
Residual Std. Error & $0.965(\mathrm{df}=853)$ & $34.569^{* * *}(\mathrm{df}=1 ; 853)$ & $35.116^{* * *}(\mathrm{df}=2 ; 852)$ \\
F Statistic & $63.821^{* * *}(\mathrm{df}=1 ; 853)$ & & ${ }^{*} \mathrm{p}<.05 ;{ }^{* *} \mathrm{p}<.01 ;{ }^{* * *} \mathrm{p}<.001$ \\
\hline \hline Note: & & &
\end{tabular}

Table 4: Mediation analysis with Objective SES

\begin{tabular}{lccc}
\hline \hline Statistic & Estimate & $95 \%$ CI Lower & $95 \%$ CI Upper \\
\hline ACME & $0.016^{* *}$ & 0.005 & 0.03 \\
ADE & $0.168^{* * *}$ & 0.097 & 0.24 \\
Total Effect & $0.185^{* * *}$ & 0.112 & 0.26 \\
Prop. Mediated & $0.090^{* *}$ & 0.0276 & 0.19 \\
\hline Note: & & ${ }^{*} \mathrm{p}<.05 ;{ }^{* *} \mathrm{p}<.01 ;{ }^{* * *} \mathrm{p}<.001$
\end{tabular}

Table 5: Mediation analysis with Subjective SES

\begin{tabular}{lccc}
\hline \hline Statistic & Estimate & $95 \%$ CI Lower & $95 \%$ CI Upper \\
\hline ACME & $0.023 *$ & 0.00491 & 0.04 \\
ADE & $0.347 * * *$ & 0.25667 & 0.44 \\
Total Effect & $0.369 * * *$ & 0.275 & 0.46 \\
Prop. Mediated & $0.062 *$ & 0.012 & 0.13 \\
\hline Note: & & ${ }^{*} \mathrm{p}<.05 ;{ }^{* *} \mathrm{p}<.01 ;{ }^{* * *} \mathrm{p}<.001$
\end{tabular}


Table 6: Mediation analysis with Treatment

\begin{tabular}{lccc}
\hline \hline Statistic & Estimate & $95 \%$ CI Lower & $95 \%$ CI Upper \\
\hline ACME & 0.001 & -0.017 & 0.02 \\
ADE & -0.060 & -0.191 & 0.07 \\
Total Effect & -0.058 & -0.189 & 0.08 \\
Prop. Mediated & -0.025 & -1.210 & 1.16 \\
\hline Note: & & ${ }^{*} \mathrm{p}<.05 ;{ }^{* *} \mathrm{p}<.01 ;{ }^{* * *} \mathrm{p}<.001$
\end{tabular}

Table 7: Descriptive statistics

\begin{tabular}{lcccc}
\hline \hline Statistic & Mean & St. Dev. & Min & Max \\
\hline Age (years) & 45.6 & 15.3 & 19 & 87 \\
Education score (1-6) & 3.7 & 1.1 & 1 & 5 \\
McArthur ladder (1-10) perceived & 4.8 & 1.7 & 1 & 10 \\
McArthur ladder correct & 3.2 & 3.0 & 1 & 10 \\
Total income & $1,157.8$ & $1,380.4$ & 0 & 10,000 \\
AUC & $20,387.9$ & $12,666.7$ & 709.0 & $49,275.5$ \\
Total social trust & -0.0 & 2.5 & -5.9 & 5.2 \\
Griskevicius score & 12.0 & 3.6 & 3 & 21 \\
\hline
\end{tabular}

Table 8: Baron and Kenny (1986) steps procedure

\begin{tabular}{lccc}
\hline \hline & \multicolumn{3}{c}{ Dependent variable: } \\
\cline { 2 - 4 } & AUC & Total Trust & Total Trust \\
& $(1)$ & $(2)$ & $(3)$ \\
\hline Objective SES & $0.096^{* *}$ & $0.136^{* * *}$ & $0.069^{*}$ \\
& $(0.030)$ & $(0.030)$ & $(0.029)$ \\
AUC & & & $0.194^{* * *}$ \\
& & & $(0.029)$ \\
& & & \\
Observations & 1,130 & 1,130 & 1,130 \\
$\mathrm{R}^{2}$ & 0.009 & 0.019 & 0.046 \\
Adjusted $\mathrm{R}^{2}$ & 0.008 & 0.018 & 0.044 \\
Residual Std. Error & $0.996(\mathrm{df}=1128)$ & $0.992(\mathrm{df}=1128)$ & $0.978(\mathrm{df}=1127)$ \\
F Statistic & $10.383^{* *}(\mathrm{df}=1 ; 1128)$ & $21.394^{* * *}(\mathrm{df}=1 ; 1128)$ & $27.230^{* * *}(\mathrm{df}=2 ; 1127)$ \\
\hline \hline Note: & & & $* \mathrm{p}<0.05 ;{ }^{* *} \mathrm{p}<0.01 ; * * * 0.001$
\end{tabular}


Table 9: Baron and Kenny (1986) steps procedure

\begin{tabular}{lccc}
\hline \hline & \multicolumn{3}{c}{ Dependent variable: } \\
\cline { 2 - 4 } & Total Trust & AUC & Total Trust \\
& $(1)$ & $(2)$ & $(3)$ \\
\hline Subjective SES & $0.260^{* * *}$ & $0.209^{* * *}$ & $0.227^{* * *}$ \\
& $(0.029)$ & $(0.029)$ & $(0.029)$ \\
AUC & & & $0.156^{* * *}$ \\
& & & $(0.029)$ \\
& & & \\
\hline Observations & 1,130 & 1,130 & 1,130 \\
$\mathrm{R}^{2}$ & 0.067 & 0.043 & 0.091 \\
Adjusted $\mathrm{R}^{2}$ & 0.067 & 0.043 & 0.089 \\
Residual Std. Error & 0.966 & 0.979 & 0.954 \\
F Statistic & $81.445^{* * *}(\mathrm{df}=1 ; 1128)$ & $51.233^{* * *}(\mathrm{df}=1 ; 1128)$ & $56.197^{* * *}(\mathrm{df}=2 ; 1127)$ \\
\hline \hline Note: & & & ${ }^{*} \mathrm{p}<0.05 ;{ }^{* *} \mathrm{p}<0.01 ;{ }^{* * *} \mathrm{p}<0.001$
\end{tabular}

Table 10: Mediation analysis with Objective SES

\begin{tabular}{lccc}
\hline \hline Statistic & Estimate & $95 \%$ CI Lower & $95 \%$ CI Upper \\
\hline ACME & $0.037 * * *$ & 0.020 & 0.06 \\
ADE & $0.097^{*}$ & 0.016 & 0.18 \\
Total Effect & $0.134^{* *}$ & 0.053 & 0.22 \\
Prop. Mediated & $0.277^{* *}$ & 0.128 & 0.71 \\
\hline Note: & & ${ }^{*} \mathrm{p}<.05 ;{ }^{* *} \mathrm{p}<.01 ;{ }^{* * *} \mathrm{p}<.001$
\end{tabular}

Table 11: Mediation analysis with Subjective SES

\begin{tabular}{lccc}
\hline \hline Statistic & Estimate & $95 \%$ CI Lower & $95 \%$ CI Upper \\
\hline ACME & $0.047^{* * *}$ & 0.0270 & 0.07 \\
ADE & $0.325^{* * *}$ & 0.2352 & 0.41 \\
Total Effect & $0.371^{* * *}$ & 0.283 & 0.46 \\
Prop. Mediated & $0.126^{* * *}$ & 0.0704 & 0.20 \\
\hline Note: & & ${ }^{*} \mathrm{p}<.05 ;{ }^{* *} \mathrm{p}<.01 ;{ }^{* * *} \mathrm{p}<.001$
\end{tabular}




\section{Acknowledgment}

We thank Rita Abdel Sater for her help during the preparation of the preregistration, especially for the power analysis of study 1 .

\section{Conflicts of interest}

None.

\section{Funding}

This study was supported by the Institut d'Etudes Cognitives (ANR-10IDEX-0001-02 FrontCog), the Institut national de la santé et de la recherche médicale (INSERM).

\section{References}

Adams, J. (June 2009). "[Commentary] TIME FOR A CHANGE OF PERSPECTIVE ON BEHAVIOUR CHANGE INTERVENTIONS?" en. In: Addiction 104.6, pp. 1025-1026. ISSN: 09652140, 13600443. DOI: 10.1111/ j.1360-0443.2009.02620.x.

Adams, J. and D. Nettle (Feb. 2009). "Time perspective, personality and smoking, body mass, and physical activity: An empirical study". en. In: British Journal of Health Psychology 14.1, pp. 83-105. ISSN: 1359107X. DOI: $10.1348 / 135910708 X 299664$.

Adler, N. E. et al. (2000). "Relationship of Subjective and Objective Social Status With Psychological and Physiological Functioning: Preliminary Data in Healthy White Women". en. In: p. 8.

Aguinis, H., J. R. Edwards, and K. J. Bradley (Oct. 2017). "Improving Our Understanding of Moderation and Mediation in Strategic Management Research". en. In: Organizational Research Methods 20.4, pp. 665-685. ISSN: 1094-4281, 1552-7425. DOI: 10.1177/1094428115627498.

Albanese, G. and G. de Blasio (Nov. 2014). "Who trusts others more? A cross-European study". en. In: Empirica 41.4, pp. 803-820. ISSN: 03408744, 1573-6911. DOI: 10.1007/s10663-013-9238-7.

Albanese, G., G. de Blasio, and P. Sestito (Dec. 2017). "Trust, risk and time preferences: evidence from survey data". en. In: International Review of Economics 64.4, pp. 367-388. ISSN: 1865-1704, 1863-4613. DOI: 10.1007/ s12232-017-0282-7. 
Alesina, A. and E. La Ferrara (Aug. 2002). "Who trusts others?" en. In: Journal of Public Economics 85.2, pp. 207-234. ISSN: 00472727. DOI: 10. 1016/S0047-2727(01)00084-6.

Balliet, D. and P. A. M. Van Lange (2013). "Trust, conflict, and cooperation: A meta-analysis." en. In: Psychological Bulletin 139.5, pp. 1090-1112. ISSN: 1939-1455, 0033-2909. DOI: 10.1037/a0030939.

Baron, R. M. and D. A. Kenny (1986). "The Moderator-Mediator Variable Distinction in Social Psychological Research: Conceptual, Strategic, and Statistical Considerations". en. In: p. 10.

Bickel, W. K. et al. (Sept. 2016). "Stuck in Time: Negative Income Shock Constricts the Temporal Window of Valuation Spanning the Future and the Past". en. In: PLOS ONE 11.9. Ed. by P. G. Roma, e0163051. ISSN: 1932-6203. DOI: 10.1371/journal . pone.0163051.

Bjørnskov, C. (Jan. 2017). "Social Trust and Economic Growth". en. In: p. 37.

Bloom, H. S. (2008). "The Core Analytics of Randomized Experiments for Social Research". en. In: The SAGE Handbook of Social Research Methods. 1 Oliver's Yard, 55 City Road, London EC1Y 1SP United Kingdom: SAGE Publications Ltd, pp. 115-133. ISBN: 978-1-84860-730-9 978-1-84860-8429. DOI: $10.4135 / 9781446212165 . n 9$.

Brandt, M. J., G. Wetherell, and P. J. Henry (Dec. 2015). "Changes in Income Predict Change in Social Trust: A Longitudinal Analysis: Socioeconomic Status and Trust". en. In: Political Psychology 36.6, pp. 761-768. ISSN: 0162895X. DOI: $10.1111 /$ pops. 12228.

Brown, W. T. and J. M. Jones (May 2004). "The Substance of Things Hoped for: a Study of the Future Orientation, Minority Status Perceptions, Academic Engagement, and Academic Performance of Black High School Students". en. In: Journal of Black Psychology 30.2, pp. 248-273. ISSN: 0095-7984, 1552-4558. DOI: 10.1177/0095798403260727.

Bublitz, E. (2017). "Misperceptions of income distributions: Cross-country evidence from a randomized survey experiment". en. In: p. 52.

Card, D. et al. (Oct. 2012). "Inequality at Work: The Effect of Peer Salaries on Job Satisfaction". en. In: American Economic Review 102.6, pp. 29813003. ISSN: 0002-8282. DOI: 10.1257/aer.102.6.2981.

Chabris, C. F. et al. (Dec. 2008). "Individual laboratory-measured discount rates predict field behavior". en. In: Journal of Risk and Uncertainty 37.23, pp. 237-269. ISSN: 0895-5646, 1573-0476. DOI: 10.1007/s11166-0089053-x.

Cruces, G., R. P. Truglia, and M. Tetaz (May 2011). "Biased Perceptions of Income Distribution and Preferences for Redistribution: Evidence from a Survey Experiment". en. In: p. 38. 
Daniel, T. O. et al. (Aug. 2015). "Episodic future thinking reduces delay discounting and energy intake in children". en. In: Eating Behaviors 18, pp. 20-24. ISSN: 14710153. DOI: 10.1016/j . eatbeh.2015.03.006.

Djimeu, E. W. and D.-G. Houndolo (Oct. 2016). "Power calculation for causal inference in social science: sample size and minimum detectable effect determination". en. In: Journal of Development Effectiveness 8.4, pp. 508527. ISSN: 1943-9342, 1943-9407. DOI: 10.1080/19439342 .2016.1244555.

Falk, A. et al. (2015). "The Nature and Predictive Power of Preferences: Global Evidence". en. In: SSRN Electronic Journal. ISSN: 1556-5068. DOI: 10.2139/ssrn. 2691910.

Fernández-Albertos, J. and A. Kuo (Jan. 2018). "Income Perception, Information, and Progressive Taxation: Evidence from a Survey Experiment". en. In: Political Science Research and Methods 6.1, pp. 83-110. ISSN: 2049-8470, 2049-8489. DOI: 10.1017/psrm.2015.73.

Frye, C. C. et al. (Jan. 2016). "Measuring Delay Discounting in Humans Using an Adjusting Amount Task". en. In: Journal of Visualized Experiments 107, p. 53584. ISSN: 1940-087X. DOI: 10.3791/53584.

Galizzi, M. M. and D. Navarro-Martinez (Mar. 2019). "On the External Validity of Social Preference Games: A Systematic Lab-Field Study". en. In: Management Science 65.3, pp. 976-1002. ISSN: 0025-1909, 1526-5501. DOI: $10.1287 / \mathrm{mnsc} .2017 .2908$.

Green, L. et al. (1996). "Temporal discounting in choice between delayed rewards: The role of age and income." en. In: Psychology and Aging 11.1, pp. 79-84. ISSN: 1939-1498, 0882-7974. DOI: 10.1037/0882-7974.11.1. 79.

Griskevicius, V. et al. (Feb. 2013). "When the Economy Falters, Do People Spend or Save? Responses to Resource Scarcity Depend on Childhood Environments". en. In: Psychological Science 24.2, pp. 197-205. ISSN: 0956-7976, 1467-9280. DOI: 10.1177/0956797612451471.

Haisley, E., R. Mostafa, and G. Loewenstein (July 2008). "Subjective relative income and lottery ticket purchases". en. In: Journal of Behavioral Decision Making 21.3, pp. 283-295. ISSN: 08943257, 10990771. DOI: 10.1002/bdm. 588.

Hardisty, D. J. et al. (2013). "How to measure time preferences: An experimental comparison of three methods". en. In: Judgment and Decision Making 8.3, p. 16 .

Harrison, G. W., M. I. Lau, and M. B. Williams (Nov. 2002). "Estimating Individual Discount Rates in Denmark: A Field Experiment". en. In: American Economic Review 92.5, pp. 1606-1617. ISSN: 0002-8282. DOI: $10.1257 / 000282802762024674$. 
Hauser, O. P. and M. I. Norton (Dec. 2017). "(Mis)perceptions of inequality". en. In: Current Opinion in Psychology 18, pp. 21-25. ISSN: 2352250X. DOI: 10.1016/j . copsyc . 2017.07.024.

Haushofer, J., D. Schunk, and E. Fehr (Sept. 2013). "Negative Income Shocks Increase Discount Rates". en. In: p. 28.

Hilbig, B. E. (July 2009). "Sad, thus true: Negativity bias in judgments of truth". en. In: Journal of Experimental Social Psychology 45.4, pp. 983986. ISSN: 00221031. DOI: 10.1016/j.jesp. 2009.04.012.

Hörl, M. et al. (Sept. 2016). "Early-Life Circumstances Predict Measures of Trust among Adults: Evidence from Hunger Episodes in Post-War Germany". en. In: The Scandinavian Journal of Economics 122.1, pp. 280305. ISSN: 0347-0520, 1467-9442. DOI: 10.1111/s joe.12329.

Howlett, E., J. Kees, and E. Kemp (June 2008). "The Role of Self-Regulation, Future Orientation, and Financial Knowledge in Long-Term Financial Decisions". en. In: Journal of Consumer Affairs 42.2, pp. 223-242. ISSN: 00220078. DOI: $10.1111 / \mathrm{j} .1745-6606.2008 .00106 . \mathrm{x}$.

Jachimowicz, J. M. et al. (May 2017). "Community trust reduces myopic decisions of low-income individuals". en. In: Proceedings of the National Academy of Sciences 114.21, pp. 5401-5406. ISSN: 0027-8424, 1091-6490. DOI: $10.1073 /$ pnas. 1617395114 .

Karadja, M., J. Mollerstrom, and D. Seim (Oct. 2014). "George Mason University Department of Economics Working Paper No. 15-10". en. In: p. 48.

Knack, S. and P. Keefer (Nov. 1997). "Does Social Capital Have an Economic Payoff? A Cross-Country Investigation". en. In: The Quarterly Journal of Economics 112.4, pp. 1251-1288. ISSN: 0033-5533, 1531-4650. DOI: 10. $1162 / 003355300555475$.

Kuziemko, I. et al. (Mar. 2013). "How Elastic Are Preferences for Redistribution? Evidence from Randomized Survey Experiments". en. In: p. 77.

Laury, S. K. and L. O. Taylor (2008). "Altruism spillovers: Are behaviors in context-free experiments predictive of altruism toward a naturally occurring public good?" In: Journal of Economic Behavior $\&$ Organization 65.1, pp. 9-29.

Lee, N. C. et al. (2013). "Age and educational track influence adolescent discounting of delayed rewards". en. In: Frontiers in Psychology 4. ISSN: 1664-1078. DOI: $10.3389 /$ fpsyg . 2013.00993.

Liu, L. et al. (Nov. 2013). "The Value of Emotion: How Does Episodic Prospection Modulate Delay Discounting?" en. In: PLoS ONE 8.11. Ed. by H. Rao, e81717. ISSN: 1932-6203. DOI: 10 . 1371 / journal . pone . 0081717.

Mannemar Sønderskov, K. (Feb. 2011). "Explaining large- $N$ cooperation: Generalized social trust and the social exchange heuristic". en. In: $R a$ - 
tionality and Society 23.1, pp. 51-74. ISSN: 1043-4631, 1461-7358. DOI: 10.1177/1043463110396058.

Mazur, J. E. (1987). "An adjusting procedure for studying delayed reinforcement". In:

Meier, S. and C. Sprenger (May 2009). "Present-Biased Preferences and Credit Card Borrowing". en. In: American Economic Journal: Applied Economics 2.1, pp. 193-210. ISSN: 1945-7782, 1945-7790. DOI: 10.1257/ app.2.1.193.

Mell, H. et al. (Mar. 2020). Early life adversity is associated with diminished social trust in adults. en. preprint. PsyArXiv. DOI: 10.31234/osf .io/ $43 \mathrm{q} 8 \mathrm{z}$.

Mellis, A. M. et al. (Oct. 2018). "Less is more: Negative income shock increases immediate preference in cross commodity discounting and food demand". en. In: Appetite 129, pp. 155-161. ISSN: 01956663. DOI: 10 . 1016/j . appet. 2018.06.032.

Micklewright, J. and S. V. Schnepf (Apr. 2010). "How reliable are income data collected with a single question?" en. In: Journal of the Royal Statistical Society: Series A (Statistics in Society) 173.2, pp. 409-429. ISSN: 09641998, 1467985X. DOI: 10.1111/j.1467-985X.2009.00632.x.

Miller, T. L. et al. (2007). "Mediation in Strategic Management Research: Conceptual Beginnings, Current Application, and Future Recommendations". en. In: Research Methodology in Strategy and Management. Vol. 4. Bingley: Emerald (MCB UP ), pp. 295-318. ISBN: 978-0-7623-1404-1. DOI: 10.1016/S1479-8387(07)04010-6.

Nguyen, Q., M.-C. Villeval, and H. Xu (2012). "Trust and Trustworthiness Under the Prospect Theory: A Field Experiment in Vietnam". en. In: SSRN Electronic Journal. ISSN: 1556-5068. DOI: 10.2139/ssrn. 2144628.

Norton, M. I. and D. Ariely (Jan. 2011). "Building a Better America-One Wealth Quintile at a Time". en. In: Perspectives on Psychological Science 6.1, pp. 9-12. ISSN: 1745-6916, 1745-6924. DOI: 10.1177/ 1745691610393524.

Norton, M. I. et al. (Dec. 2014). "The Not-So-Common-Wealth of Australia: Evidence for a Cross-Cultural Desire for a More Equal Distribution of Wealth: The Not-So-Common-Wealth of Australia". en. In: Analyses of Social Issues and Public Policy 14.1, pp. 339-351. ISSN: 15297489. DOI: 10.1111/asap. 12058.

Pepper, G. V. and D. Nettle (2017). "The behavioural constellation of deprivation: Causes and consequences". en. In: Behavioral and Brain Sciences 40, e314. ISSN: 0140-525X, 1469-1825. DOI: 10 . 1017 / S0140525X1600234X. 
Petersen, M. B. and L. Aarøe (Nov. 2015). "Birth Weight and Social Trust in Adulthood: Evidence for Early Calibration of Social Cognition". en. In: Psychological Science 26.11, pp. 1681-1692. ISSN: 0956-7976, 1467-9280. DOI: $10.1177 / 0956797615595622$.

Rachlin, H. (May 2006). "NOTES ON DISCOUNTING". en. In: Journal of the Experimental Analysis of Behavior 85.3, pp. 425-435. ISSN: 00225002. DOI: $10.1901 /$ jeab.2006.85-05.

Reimers, S. et al. (Dec. 2009). "Associations between a one-shot delay discounting measure and age, income, education and real-world impulsive behavior". en. In: Personality and Individual Differences 47.8, pp. 973978. ISSN: 01918869. DOI: 10.1016/j.paid.2009.07.026.

Rönnerstrand, B. (Dec. 2013). "Social capital and immunisation against the 2009 A(H1N1) pandemic in Sweden". en. In: Scandinavian Journal of Public Health 41.8, pp. 853-859. ISSN: 1403-4948, 1651-1905. DOI: 10 . $1177 / 1403494813494975$.

Schoemann, A. M., A. J. Boulton, and S. D. Short (May 2017). "Determining Power and Sample Size for Simple and Complex Mediation Models". en. In: Social Psychological and Personality Science 8.4, pp. 379-386. ISSN: 1948-5506, 1948-5514. DOI: 10.1177/1948550617715068.

Stamos, A., E. Altsitsiadis, and S. Dewitte (July 2019). "Investigating the effect of childhood socioeconomic background on interpersonal trust: Lower childhood socioeconomic status predicts lower levels of trust". en. In: Personality and Individual Differences 145, pp. 19-25. ISSN: 01918869. DOI: 10.1016/j.paid.2019.03.011.

Sze, Y. Y. et al. (July 2017). "Bleak Present, Bright Future: Online Episodic Future Thinking, Scarcity, Delay Discounting, and Food Demand". en. In: Clinical Psychological Science 5.4, pp. 683-697. ISSN: 2167-7026, 21677034. DOI: $10.1177 / 2167702617696511$.

Tingley, D. et al. (2014). "mediation : R Package for Causal Mediation Analysis". en. In: Journal of Statistical Software 59.5. ISSN: 1548-7660. DOI: 10.18637/jss.v059.i05.

Voors, M. et al. (Mar. 2012). "Exploring whether behavior in context-free experiments is predictive of behavior in the field: Evidence from lab and field experiments in rural Sierra Leone". en. In: Economics Letters 114.3, pp. 308-311. ISSN: 01651765. DOI: 10.1016/j.econlet.2011.10.016. 